J. Perinat. Med. 17 (1989) 411

\title{
Evaluation of fetal heart rate artifacts, hemodynamics and digoxin treatment in fetal tachyarrhythmia by Doppler measurement of fetal blood flow - case report of a pre-excitation syndrome
}

\author{
Markus Gonser, Johannes Dietl, Karlheinz Pfeiffer, and Jean-Pierre Clees \\ Department of Obstetrics and Gynecology, University Hospital Tübingen, West \\ Germany
}

\section{Introduction}

A fetal cardiac arrthythmia may cause uninterpretable or even misleading fetal heart rate (FHR) tracings with unreliable signs of fetal distress [1]. Therefore evaluation of such artifacts and the haemodynamic relevance of a fetal arrhythmia by alternative methods [9] is necessary for management and therapy. Doppler measurement of fetal blood flow has been reported to be useful for hemodynamic assessment of fetal cardiac arrythmias [9, 10], for detection of imminent heart failure prior to fetal hydrops and for monitoring of therpaeutic effects of therapy [11].

\section{Material and methods}

External indirect FHR-monitoring during pregnancy and internal direct FHR-monitoring from the fetal scalp during labor and delivery was performed with a comerical fetal monitoring system with autocorrelation technique (HP 8040 Cardiotocograph, Hewlett-Packard, FRG). For the recording of a tachycardic FHR, HewlettPackard (Böblingen, FRG) supplied an especially constructed HP 8041A cardiotocograph prototype.

FHR records from both systems were checked by cw (continuous wave)-Doppler blood flow measurements in umbilical artery (Doptek Spectrum Analyser 9000 with built-in $4 \mathrm{MHz}$ cwDoppler, Foptek Ltd, GB).

Blood flow in the fetal descending aorta was measured with combined real-time and pulsed Doppler ultrasound (Kretz Combison 320-5:

\section{Curriculum vitae}

MARKUS J. GonSER, M.D., was born in 1953 in Horb-Altheim, West Germany. He studied mathematics and physics, from 1973 to 1977, medicine, from 1977 to 1984 , and in 1984, he graduated from the Faculty of Medicine at the University of Tübingen. From 1984 to 1985, he did industrial re-

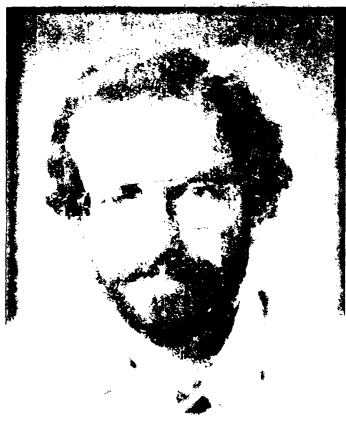
search fellowship in pharmacokinetics from the Boehringer Ingelheim Fonds, Foundation for basic research in medical sciences. Since 1985, he is specializing in obstetrics and gynecology at the University of Tübingen.

$5 \mathrm{MHz}$ Sector Ultrasound Scanner with built in $2.2 \mathrm{MHz}$ pulsed Doppler, Duplex system, KretzTechnik, Austria); with real-time display of "full" spectral Doppler sonogram (pulse repetition frequency PRF: $6.25 \mathrm{MHz}$, wall motion filter WMF: $125 \mathrm{~Hz}$; adjustments fixed by M. G.) and off-line estimate of mean velocity (intensityweighted spectral and temporal average). Fetal size and vessel diameter were measured on frozen ultrasound image from the same system.

\section{Clinical information and results}

A 28-year-old healthy woman was referred at 30 weeks of gestation because of fetal tachycardiabradycardia-arrthythmia. Apart from a hydropic placenta, ultrasound examination showed nor- 


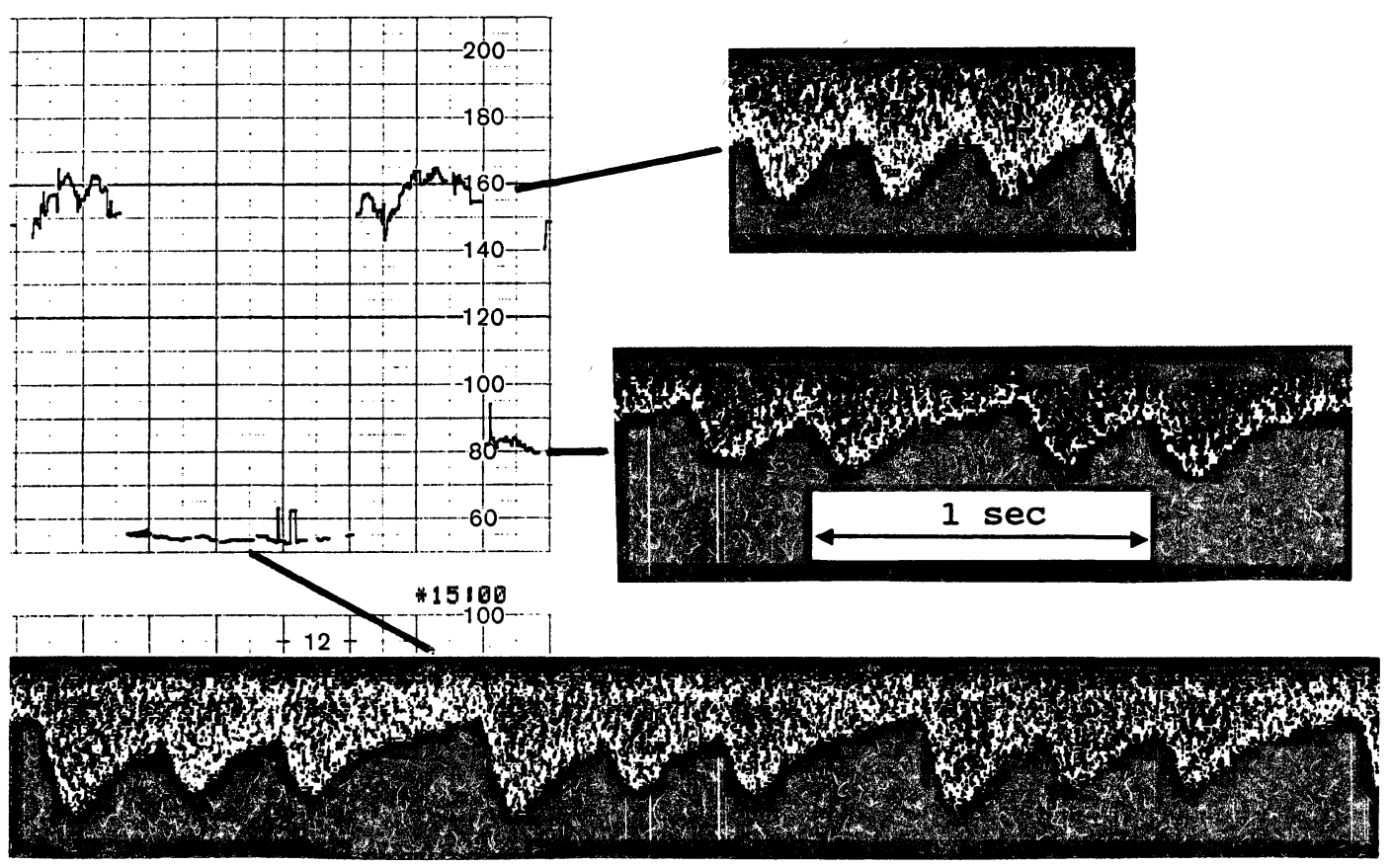

Figure 1. External FHR record in fetal arrhythmia with paroxysmal bigeminal and trigeminal pulse. Check of the questionable tracings with cw-Doppler blood flow measurement in umbilical artery (pseudobradycardia).

mal fetal and cardiac anatomy, but with arrhythmia in fetal real-time and M-mode echocardiography [7, 8]. The obtained FHR-record was irregular, showing alternating periods of severe bradycardia. Checking this questionable tracing by cw-Doppler assessment of umbilical artery blood flow revealed periods of bigeminal and trigeminal pulse with a numerically normal heart rate (figure 1). Obviously the fetal monitoring system was triggered by one of two or three pulsations respectively and thus demonstrated pseudobradycardia.

During the next four hours, the FHR turned to regular tachycardia by ascultation, but conventional FHR-monitor concealed fetal tachycradia and mimicked falsely normal FHR. A second FHR-monitor prototype, designed especially for fetal tachycardia, recorded FHR correctly, with a mean hart rate of $240 \mathrm{bpm}$ as demonstrated with cw-Doppler (figure 2). Oral transplacental digitalization was started (beta-acetyldigoxin, $3 \times 0.2 \mathrm{mg} / \mathrm{d}$ ) [4] to prevent recurrent tachycardia and worsening of fetal heart failure [7, 11 15]. Transplacental cardioversion was not achieved definitively, however FHR was basically normal during treatment.
Between 32 and 34 gestational weeks, the patient discontinued digitalis intake against medical advice, and a temporary pericardial effusion, between 34 and 36 weeks, indicated subsequent worstening of fetal cardiac failure [2]. Serial pulsed Doppler measurements of fetal aortic blood flow $[3,9,10]$ were performed at 30,32 , 33,35 and 36 weeks of gestation. Thus imminent fetal heart failure was recognized after discontinued medication and before pericardial effusion occured. Furthermore, improvement of fetal cardiac performance secondary to restarting the medication and prior to resalution of pericardial effusion was recognized also (figure 3).

During spontaneous labor and delivery, at 37 weeks of gestation, fetal arrhythmia with bigeminal pulse recurred, and again internal FHR monitoring from the fetal scalp showed pseudobradycardia; however, the FHR recording was correct when "logic" was switched off (figure 4). The nonhydropic male infant weighed $4150 \mathrm{~g}$ and demonstrated minimal signs of neonatal distress (Apgar score 5 and 7 at 1 and $5 \mathrm{~min}$ respectively) with delayed onset of respiration and bradycardia, although the $\mathrm{pH}$ of umbilical cord blood was normal (art: 7.32, ven: 7.39). 

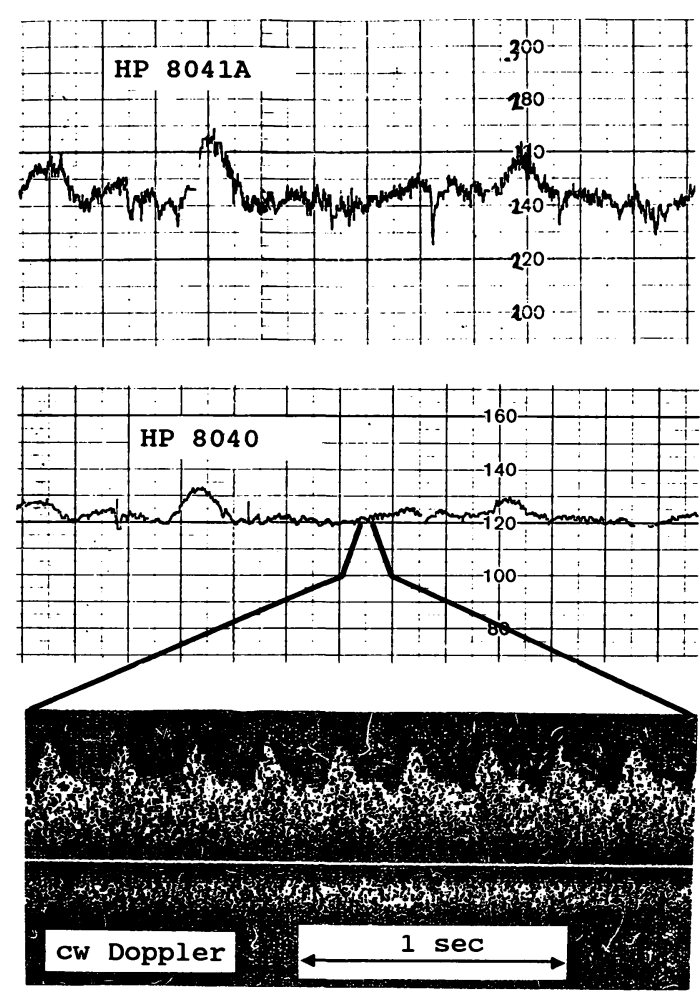

Figure 2. Fetal tachycardia with two-to-one block in conventional fetal monitor (CTG artifact). Top: External prototype FHR-monitor $\left({ }^{*}\right)$, showiing a correct trace. Middle: External convention FHR-monitor with misleading "normal" tracing, as a result of "halving" true FHR [5]. Bottom: Check of FHR with cw-Doppler assessment of umbilical artery blood flow: $240 \mathrm{pbm}$. (*) Prototype supplied by Hewlett-Packard, Böblingen, FRG.

Postpartum echocardiography confirmed normal heart anatomy and ECG showed pre-exitation with delta-waves [14], but no signs of digitalis intoxication. Thus a Wolff-Parkinson-White syndrome was diagnosed and continuation of digoxin treatment was indicated $[6,12]$. At six months of life, treatment was stopped and further examinations revealed normal development and normal sinus rhythm (Dept. of Pediatrics, Div. of Pediatric Cardiology, University Hospital, Tübingen).

\section{Discussion}

In fetal monitoring systems, preprocessing of incoming signals according to certain preset lim-
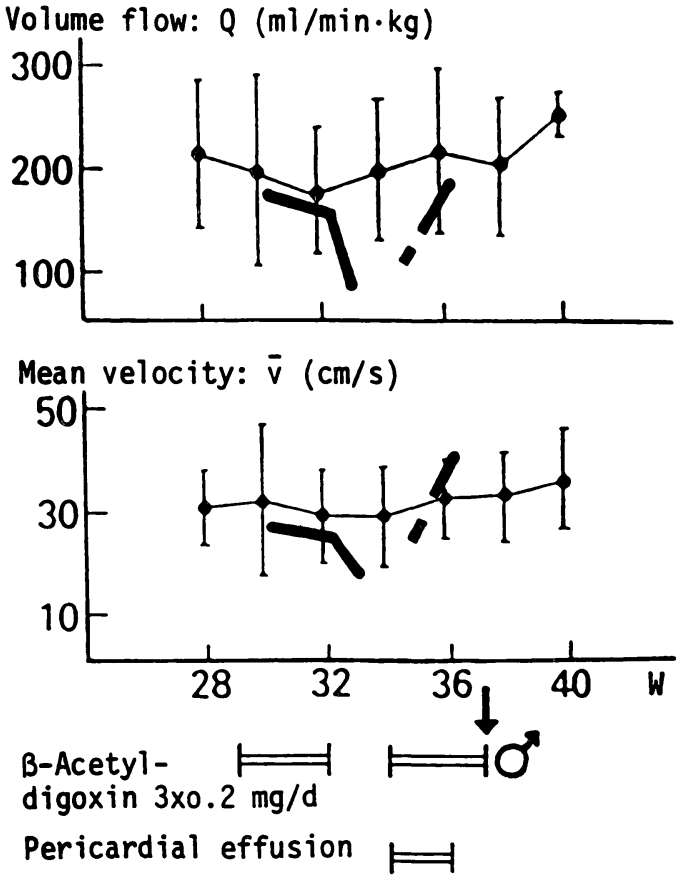

Figure 3. Blood flow in fetal descending aorta: Recognition of impairment and improvement of fetal cardiac performance prior to evolution and prior to resolution of pericardial effusion. This effusion appeared after discontinued digitalis intake. Spontaneously delivery after 37 weeds of gestation, $4150 \mathrm{~g}$, Apgar 5/7/9, pH A: 7.32; V: 7.39 .

(Nomogram after ERSKINE RLA and RITCHIE JWK [3]).

its, such as maximal FHR range or maximal FHR changes ("logic"), and autocorrelation technique allow indirect and automatical FHR detection and recognition, in spite of minimal maternal or fetal movements. These technical approaches can obscure FHR monitor and falsely normal FHR is mimicked [5, 7, 8, 13]. In this case, pre-excitation with bi- or trigeminal pulse caused severe tachycardia, but on the fetal monitor appeared a "normal" heart rate tracing (figure 2); or during the preceding and intrapartum periods of bi- and trigeminal pulse FHR was numerically normal, but a severe bradycardia was recorded (figures 1 , and 4).

$\mathrm{Cw}$-Doppler assessment of umbilical artery blood flow was a suitable method to check questionable FHR recordings quickly. With serial pulsed Doppler measurements of fetal aortic blood flow, it was possible, first to recognize 

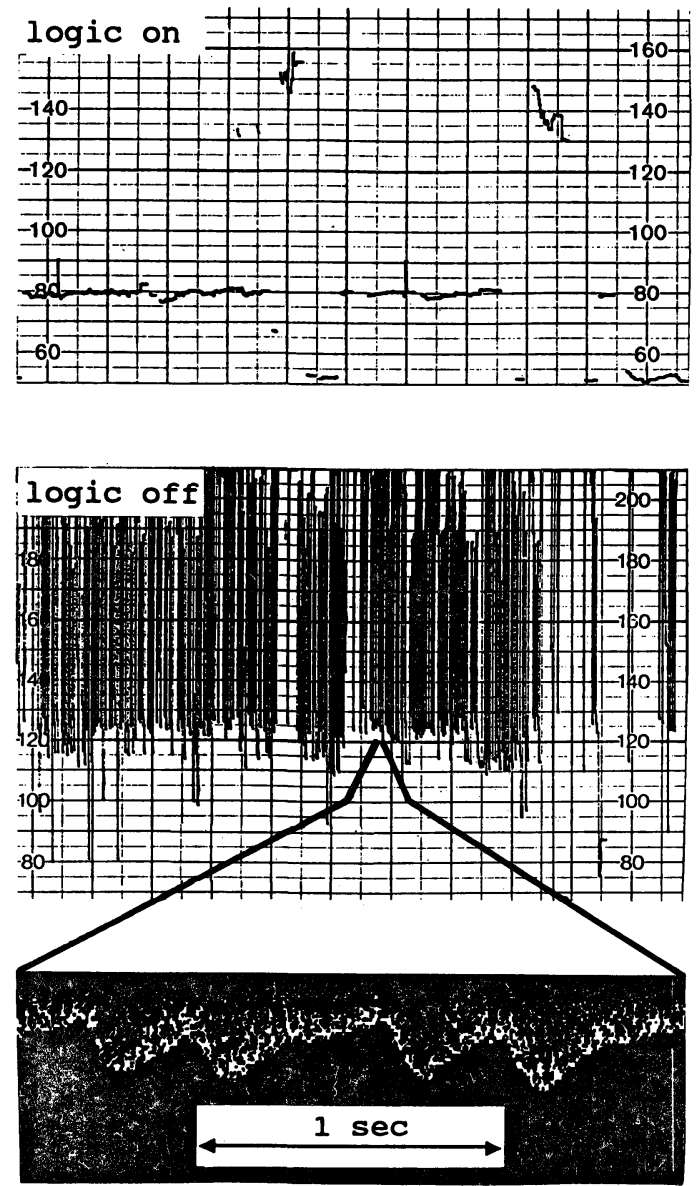

\begin{abstract}
A fetal cardiac arrhythmia may cause misleading fetal heart rate tracings with unreliable signs of fetal distress. Assessment of such artifacts and of the hemodynamic relevance of a fetal arrhythmia by alternative methods is necessary for management and therapy.
\end{abstract}

A 28-year-old healthy woman was referred at 30 weeks of gestation because of fetal tachy-brady-arrhythmia, but cw-Doppler assessment of umbilical artery blood flow revealed periods of pseudobradycardia during bigeminal and trigeminal fetal pulse. FHR turned to regular tachycardia, and transplacental digitalization was started. Between 32 and 34 weeks the patient discontinued her digitalis intake, and a fetal pericardial effusion indicated subsequent cardiac failure. Serial impairment of fetal cardiac performance after discontinued digitialis intake and prior to development of pericardial effusion - and second, to recognize cardiac improvement after restarting medication and prior to resolving the effusion (figure 3).

New monitoring developments and Doppler measurement of fetal blood flow may be of help to verify fetal arrthythmia and to quantify their hemodynamic implications $[9,10]$. An immiment heart failure can be detected before signs of fetal hydrops occur and the therapeutic effect of medication can be monitored by serial measurements of fetal blood flow [11].

Figure 4. Pseudobradycardia in fetal bigeminal pulse. Top: Mimicked bradycardia in conventional external fetal monitoring. Middle: True fetal arrhythmia in conventional internal fetal monitoring and "logic off". Bottom: Verification of fetal bigeminal pulse by Doppler assessment of umbilical artery blood flow.

pulsed Doppler measurements of fetal aortic blood flow were performed and imminent heart failure was recognized after the digitalis was discontinued and before a pericardial effusion occured. Furthermore, improvement of fetal cardiac performance secondary to restarting digitalis and also prior to resolution of the pericardial effusion. After spontaneous delivery a Wolff-Parkinson-White syndrome was diagnosed, and continuation of digoxin treatment was indicated.

Cw-Doppler assessment of umbilical artery blood flow was a suitable method to evaluate questionable FHR recordings, and pulsed Doppler allowed monitoring of the therapeutic effect of transplacental digitalization by serial measurements of fetal aortic blood flow.

Keywords: Bigeminal pulse, CTG artifacts, Doppler ultrasound, fetal arrhythmia, fetal blood flow, fetal digitalization, pseudobradycardia, Wolff-Parkinson-White syndrome. 


\section{Zusammenfassung}

CTG-Artefakte, Hämodynamik und Digitalisierung bei fetalen Tachyarrhythmien - dopplersonographische Überwachung am Beispiel eines Präexzitationssyndroms Eine fetale cardiale Arrhythmie kann nicht interpretierbare oder irreführende Aufzeichnungen der fetalen Herzfrequenz (FHF) verursachen. Daher ist die Untersuchung solcher CTG-Artefakte [1] und der hämodynamischen Relevanz einer aktuellen fetalen Arrhythmie durch alternative Methoden [9] im Hinblick auf Management und Therapie notwendig.

Die externe CTG-Überwachung erfolgte mit einem herkömmlichen Autokorrelationscardiotokographen (HP 8040). Die FHF-Aufzeichnungen wurden durch dopplersonographische Strömungsmessungen in der Umbilikalarterie überprüft (Doptek Spectrum Analyser 9000 mit eingebautem $4 \mathrm{MHz}$ cw-Doppler). Die Durchblutung in der absteigenden fetalen Aorta wurde mit kombinierter Real-Time und Pulsdoppler-Sonographie gemessen (Kretz Combison 320-5, DuplexSystem).

Uns wurde eine 28jährige gesunde Frau in der 30. Schwangerschaftswoche wegen fetaler Tachy-/Bradyarrhythmien überwiesen. Die FHF-Aufzeichnungen waren irregulär, alternierend traten Phasen mit schwerer Bradykardie auf. Die zugehörigen Dopplersonogramme der Umbilikalarterie zeigten jedoch Perioden mit Bigeminus und Trigeminus, wobei die Herzfrequenz numerisch normal war (Abb. 1 u. 4). Offensichtlich wurden im CTG nur eine von 2 oder 3 Pulsationen erkannt und es zeigte daher eine Pseudobradykardie. Während der nächsten Stunden wechselte die FHF zu einer regelmäßigen Tachykardie mit einer mittleren
Frequenz von $240 \mathrm{spm}$, und wir begannen mit einer oralen transplazentaren Digitalisierung [15]. Zwischen 32 und 34 Wochen unterbrach die Patientin die Digitaliseinnahme und zwischen 34 und 36 Wochen trat zeitweilig ein Perikarderguß als Hinweis auf eine manifeste Herzinsuffizienz auf [2]. Die Durchblutungsmessungen in der fetalen Aorta ließen eine Verschlechterung der Herzleistung nach Absetzen der Medikation und vor Auftreten des Perikardergusses erkennen. Im weiteren Verlauf war eine Verbesserung der Herzleistung nach Wiedereinsetzen der Medikation und vor Rückbildung des Perikardergusses nachweisbar (Abb. 3).

Nach 37 Schwangerschaftswochen kam es zur Spontangeburt eines Kindes ohne Hydrops. Man diagnostizierte ein Wolff-Parkinson-White-Syndrome und setzte die Digitalisbehandlung fort $[6,12]$.

Die Bearbeitung aufgenommener Signale gemäß gewisser vorgegebener Grenzen wie maximalen Bereich der FHF und maximale FHF-Änderungen ("Logik") ermöglicht eine automatische FHF-Anzeige und -Erkennung. Jedoch können mit dieser Technik FHFAufzeichnungen bei fetalen Tachyarrhythmien unklar bleiben [1]. Doppleruntersuchungen der Nabelarterien erwiesen sich als geeignete Methode, um zweifelhafte FHF-Aufzeichnungen abzuklären. Mit dem gepulsten Doppler war es möglich, eine beginnende Herzinsuffizienz vor Auftreten von Hydropszeichen zu erkennen und den therapeutischen Effekt einer transplazentaren Digitalisierung durch Verlaufsmessungen der fetalen Aortendurchblutung nachzuweisen [11].

Schlüsselwörter: Bigeminus, CTG-Artefakte, Dopplersonographie, fetale Arrhythmie, fetale Digitalisierung, fetaler Blutfluß, Pseudeobradykardie, Wolff-Parkinsln-White-Syndrom.

\section{Résumé}

Evaluation des artefacts CTG, de l'hémodynamique et du traitement par digoxine dans les tachyarythmies fetales a l'aide de la mesure Doppler du FLUX sanguin fetal. A propos d'un cas de syndrome de préexitation L'arythmie cardiaque fœtale peut entraîner des enrigstrements du rythme cardiaque fœtal (RCF) ininterprétables ou même trompeurs. C'est la raison pour laquelle la mise en évidence de tels artefacts du RCF [1] et de l'hémodynamique seconaires à une arythmie fœtale en cours par des méthodes alternatives [9] est nécessaire pour la prise en charge et la thérapeutique. Le monitorage du RCF par voie externe a été réalisé à l'aide d'un système du surveillance fotale du commerce avec une technique d'auto-corrélation (cardiotocographe HP 8040). Les enregistrements du RCF ont été complétés par des mesures doppler au niveau de l'artère ombilicale (Doptek spectrum analyser 9000 avec $4 \mathrm{MHZ} \mathrm{cw}$ Doppler), et de l'aorte descendante par association d'échographie combinée en temps réel et doppler pulsé (Kretz Combison 320-5, Duplex system).

Une patiente de 29 ans, en bonne santé a été adressée à 30 semaines de gestation en raison d'une tachybrady-arythmie fotale. Le RCF étaie irrégulier, avec alternance de périodes de bradycardies majeures, mais l'examen doppler de l'artère ombilicale a mis en évidence des périodes de bigéminisme et de trigéminisme avec un rythme cardiaque fœtal numériquement normal (figures 1, 4). A l'évidence le monitorage fotal à lui seur ne reconnaît qu'une des deux ou trois pulsations respectives et met en évidence une pseudobradycardie.

Au cours des heures suivantes, le RCF évolua vers une tachycardie régulière, avec un rythme moyen de 240 $\mathrm{bpm}$, et une digitalisation transplacentaire par voie orale fut commencée [15]. Entre 32 et 34 semaines, la patiente interrompit son traitement digitalique et un épanchement péricardique temporaire, entre 34 et 36 
semaines, fut le témoin d'une défaillance cardiaque consécutive [2]. On effectua des séries de mesure doppler pulsé du flux sanguin aortique fæalal ce qui permit de reconnaître une défaillance des performances cardiaques fœtales après arrêt de la thérapeutique. En outre, l'amélioration des performances cardiaques fœtales secondaires au redémarrage de la thérapeutique et avant la disparition de l'épanchement péricardique fut mis en évidence (Figure 3).

A 37 semaines de gestation naquit un enfant nonhydropique de façon spontanée. On fit le daignostic de syndrome de Wolf-Parkinson White et la poursuite du traitement digoxique fut indiquée $[6,12]$.

Le prétraitement des signaux d'entrée selon certaines limites préétablies, telles que l'étendue maximale du CRF et les variations maximales du RCF ("logiques"), permet la détection automatique du RCF et sa reconnaissance. Mais cette technique peut masquer l'enregistrement du RCF en cas de tachy-arythmie fotale [1]. L'étude doppler du flux sanguin dans l'artère ombilicale est une méthode appropriée pour faire le bilan d'enregistrement du rythme cardiaque fotal à problème. Par doppler pulsé il est possible de reconnaître une défaillance cardiaque imminente avant l'apparition des signes d'hydrops fœtal, et de surveiller les effets thérapeutiques de la digitalisation transplacentaire par détermination en série du flux sanguin aortique fœtal [11].

Mots-clés: Artefacts CIG, arythmie fœtale, débit sanguin fœtal, digitalisation fœtale, échographie Doppler, pseudobradycradie, pulse bigéminé, syndrome de Wolf-Parkinson-White.

\section{References}

[1] Beall MH, RH Paul: Artifacts, blocks and arrythmias: confusing nonclassical heart rate tracings. Clin Obstet Gynecol 29 (1986) 83

[2] DeVore GR, RL Donnerstein, CS Kleinman, LD PlatT, JC HobBINS: Fetal echocardiography. II. The diagnosis and significance of a pericardial effusion in the fetus using real-time-directed Mmode ultrasound. Am J Obstet Gynecol 144 (1982) 693

[3] ERSKINE RLA, JWK RitchIE: Quantitative measurement of fetal blood flow using Doppler ultrasound. Br J Obstet Gynaecol 92 (1985) 600

[4] GonSER M: Transplacental digitalisation of the fetus - Proposal of loading and maintenance dose calculations based on maternal weight and creatinine clearance. Naunyn-Schmiedeberg's Arch Pharmacol 337 (1988) R4 (suppl), abstr

[5] HeRTOGs K: Supraventricular tachycardia in utero with two-to-one block in fetal monitor. Lancet i (1981) 1158

[6] JAMES TN: Cardiac conduction system: fetal and postnatal development. Am J Cardiol 25 (1970) 213

[7] Klein AM, IR Holzman, EM Austin: Fetal tachycardia prior to the development of hydrops - Attempted pharmacological cardioversion: Case report. Am J Obstet Gynecol 134 (1979) 347

[8] Kleinman CS, RL Donnerstein, CC JafFe, GR DeVore, EM WeINSTEIN, DC LyNCH, NS TALNER, RL BERKowITZ, JC HoBbINS: Fetal echocardiography. A tool for evaluation of in utero cardiac arrhythmias and monitoring of in utero therapy: analysis of 71 patients. Am J Cardiol 51 (1983) 237

[9] Lingman G, J-A Dahlström, SH Eik-Nes, K Marsal, P OHLIN, S OHRLANDER: Haemodynamic assessment of fetal heart arrhythmias. $\mathrm{Br} \mathbf{J}$ Obstet Gynecol 91 (1984) 647
[10] Marsal K, A Lindblad, G Lingman, SH Eiknes: Blood flow in the fetal descending aorta; intrinsic factors affecting fetal blood flow, i.e. fetal breathing movements and cardiac arrhythmias. Ultrasound in Med Biol 10 (1984) 339

[11] Marsal K, A Lindblad, G Lingman, P SindBERG-ERIKSEN: Fetal circulatory changes associated with cardiac arrhythmia, smoking and experimental asphyxia. In: SHELDON CD, DH EVANS, JR SALVAGE (eds.): Obstetric and neonatal blood flow. Biological Engineering Society, Conference Proceedings, Vol 2, London, 1987, pp $59-66$

[12] MoENE RJ, JP Roos: Transient Wolff-ParkinsonWhite syndrome and neonatal reciprocating tachycardia. Circulation 59 (1973) 443

[13] ReEs L, PR Vlies, J Adams: Hydrops fetalis, an unusual cause, presentation and method of diagnosis. Case report. Br J Obstet Gynaecol 87 (1980) 1169

[14] Vidaillet HJ, JC Pressley, E Henke, fE HarRELL, LD GERMAN: Familial occurence of accessory atrioventricular pathways (preexcitation syndrome). N Engl J Med 317 (1987) 65

[15] WladimirofF JW, PA Stewart: Fetal therapy. Treatment of fetal cardiac arrhythmias. Br J Hosp Med 34 (1985) 134

Received March 20, 1989. Accepted October 16, 1989.
Dr. Markus Gonser
Universitäts-Frauenklinik
Schleichstr. 4
D-7400 Tübingen
West Germany 